\title{
Unilateral Bi-portal Endoscopic Decompression via the Contralateral Approach in Asymmetric Spinal Stenosis: A Technical Note
}

\author{
Jin-Sung Kim ${ }^{1,}$, Cheol-Woong Park ${ }^{2, *}$, Yip-Kan Yeung ${ }^{3}$, Tsz-King Suen ${ }^{4}$, Su Gi Jun ${ }^{2}$, Jung-Hoon Park ${ }^{2}$ \\ ${ }^{1}$ Department of Neurosurgery, Spine Center, Seoul St. Mary's Hospital, College of Medicine, The Catholic University of Korea, Seoul, Korea \\ ${ }^{2}$ Department of Spinal Surgery, Daejeon Woori Hospital, Daejeon, Korea \\ ${ }^{3}$ Department of Orthopaedics and Traumatology, Caritas Medical Centre, Kowloon, Hong Kong \\ ${ }^{4}$ Department of Orthopaedics and Traumatology, Hong Kong Baptist Hospital, Kowloon, Hong Kong
}

We discuss the technical details and operative advantages of approaching pathologies from the contralateral side in cases of asymmetric spinal stenosis. The contralateral approach offers better manipulative freedom and a more accessible target approach along the plane of the pathology, allowing safer decompression and facet preservation; further, this approach is ergonomic for surgeons. We recommend the adoption of this approach in decompressing asymmetric spinal stenosis.

Keywords: Endoscopy; Laminectomy; Spinal stenosis

\section{Introduction}

Spinal laminectomy decompression is the most common spinal operation for patients aged $>65$ years [1]. The goal is to decompress the symptomatic neural elements without violating spinal stability. Surgical techniques have evolved over the years from open to microscopic, microendoscopic, and endoscopic surgery. Conditions, such as ligamentum flavum hypertrophy or ossification, disc degeneration, facet arthropathy, and cystic degeneration contribute to the clinical severity of spinal stenosis and increase the operative difficulties.

For asymmetric spinal stenosis, open decompression with the ipsilateral approach enables a direct and shorter route [2]. However, conditions, such as facet hypertrophy or ipsilateral spinous process deviation, may hinder ipsilateral access and necessitate partial facetectomy to expose the lateral recess. A more recent study has suggested that juxtafacet cyst decompression may be safer and more easily accessible from the contralateral side in bi-portal endoscopic surgery [3]. However, to our knowledge, no study has analyzed the potential technical advantages of this method over those of the ipsilateral approach.

This manuscript aimed to illustrate three case examples to describe the surgical techniques and highlight the advantages of unilateral bi-portal endoscopic decompression for asymmetric spinal stenosis via the contralateral approach.

Received Mar 22, 2020; Revised Jun 26, 2020; Accepted Jul 30, 2020

Corresponding author: Yip-Kan Yeung

Department of Orthopaedics and Traumatology, Caritas Medical Centre, 111 Wing Hong Street, Kowloon, Hong Kong

Tel: +852-66290407, Fax: +852-21484399, E-mail: yyk028@ha.org.hk

*These authors contributed equally to this work. 


\section{Technical Note}

\section{Steps}

\section{1) Step 1}

Preoperative templating on imaging films is done to identify the portal trajectory. The patient is under general/ epidural anesthesia and is positioned prone on a spinal frame. The lumbar spine is slightly flexed to open up the interlaminar space.

\section{2) Step 2}

The surgical site is sterilized and draped with waterproof drapes. A sectioned oxygen tube is mounted under OpSite film in a U-shaped fashion, opening up on the opposite side, to direct the water outflow away from the operating surgeon (Fig. 1A).
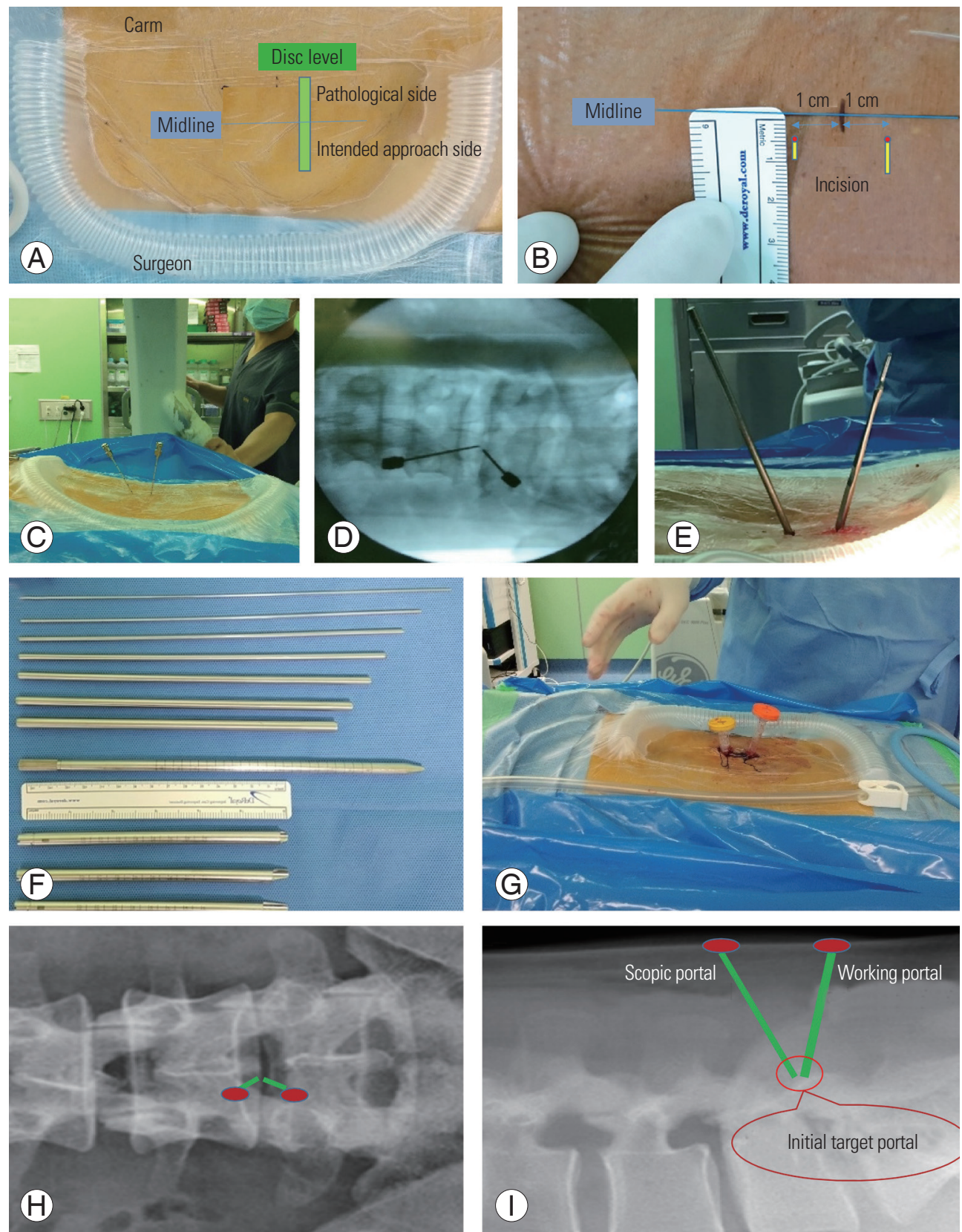

Fig. 1. Intraoperative photos of (A) surgical draping, (B) skin marking (the two red dots represent needles entry site; the yellow transverse lines indicate skin incisions), (C) spinal needles insertion, (D) fluoroscopic confirmation of needles position, (E) blunt dissection by metal rods, (F) serial dilators, (G) viewing and working cannulas, and (H, I) desired portals position in X-rays. 


\section{3) Step 3}

The midline and targeted disc level is identified on fluoroscopy (Fig. 1A). The C-arm machine and fluoroscopic screen is positioned on the opposite side to ensure that the machine does not interfere with the surgeon's standing position. The anticipated needle entry sites and incisions are marked (Fig. 1B).

\section{4) Step 4}

Two spinal needles are inserted $5 \mathrm{~mm}$ away from the midline over the planned approach side, one at $10 \mathrm{~mm}$ cranial and the other at $10 \mathrm{~mm}$ caudal to the disc level. They are triangulated towards each other till the tip ends meet. Both the needles are aimed toward the most caudal end of the spinolaminar junction of the cranial lamina under fluoroscopy (Fig. 1C, D).

\section{5) Step 5}

At the needle entry points, 5-mm and 8-mm transverse skin incisions are created for viewing (cranial) and working (caudal) the portals. Deeper incisions are made medially, crossing the muscle fascia for cannula insertion, and more superficial incisions are made laterally crossing the skin to allow only oblique instrumental manipulation to the contralateral side. Blunt metal rods are inserted toward each other to create a working space beneath the multifidus muscle (Fig. 1E).

\section{6) Step 6}

Serial dilators are inserted sequentially, and transparent cannulas are inserted over the dilators toward the spinolaminar junction. The $5-\mathrm{mm}$ viewing cannula is shaped beveled to allow gentle dural retraction by the transparent lip without obstructing the endoscopic view. The $8-\mathrm{mm}$ working cannula with threads can be screwed in or out to adjust its depth for facilitating instrument manipulation (Figs. 1F-I, 2).

\section{7) Step 7}

Water influx is connected to the metal endoscopic portal, inserted via the viewing cannula. The viewing cannula can be removed later after the viewing tract is well established. Gravity water pressure prevents intracranial hypertension and tissue edema. Smooth water outflow should be ascertained to ensure an adequate dissipation of pressure and heat away from the spinal canal. A zero-degree endoscope is inserted via the viewing portal, and a radiofrequency probe was inserted via the working portal.

\section{8) Step 8}

Starting from the spinolaminar junction, the soft tissue overlying the lower lamina of the cranial vertebra is ablated to enable bone edge exposure (Fig. 3A). A 4-mm shielded cutting burr is used for central laminotomy and contralateral sublaminoplasty until the cranial insertion
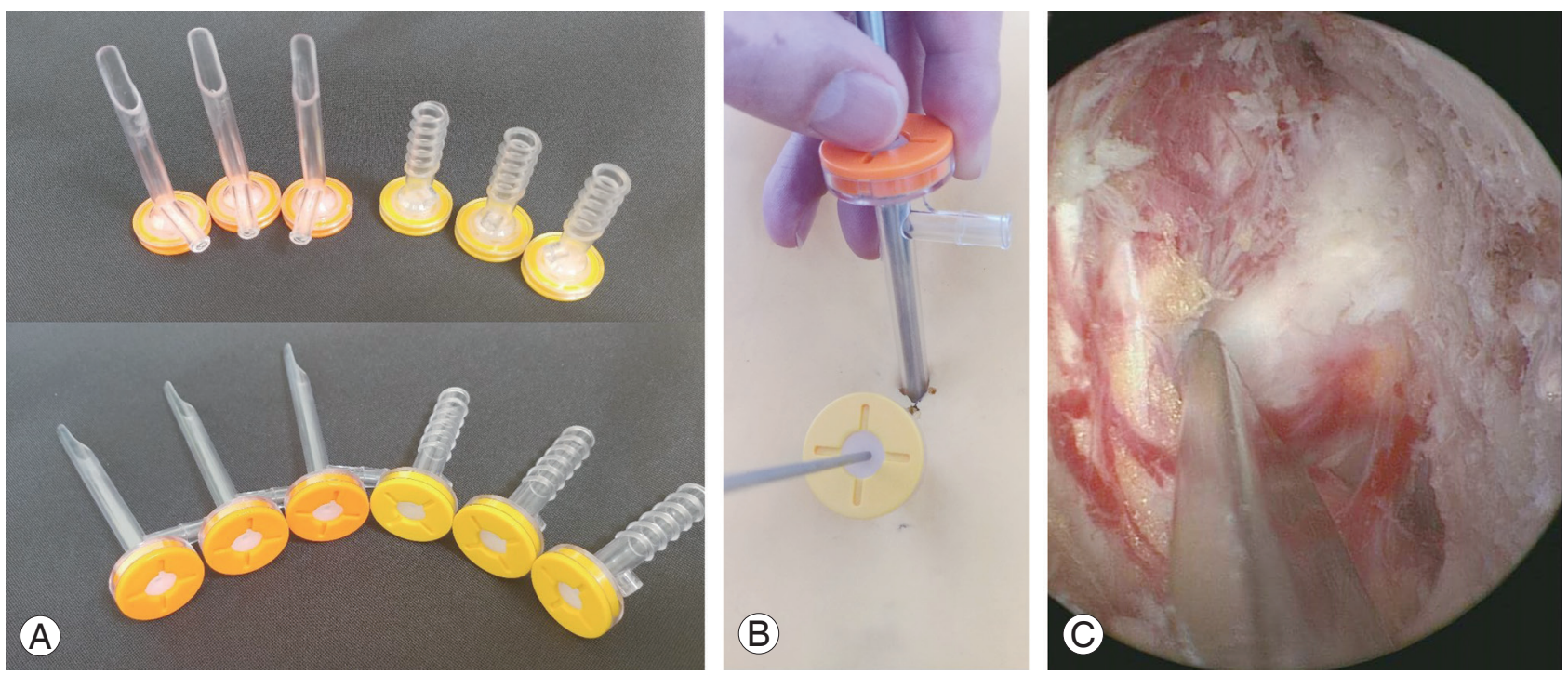

Fig. 2. (A) Beveled viewing cannulas and threaded working cannulas. (B) Insertion of scope into viewing cannula and instrument into working cannula. (C) The beveled lip of the cannula can be rotated for facilitating gentle dural retraction. 

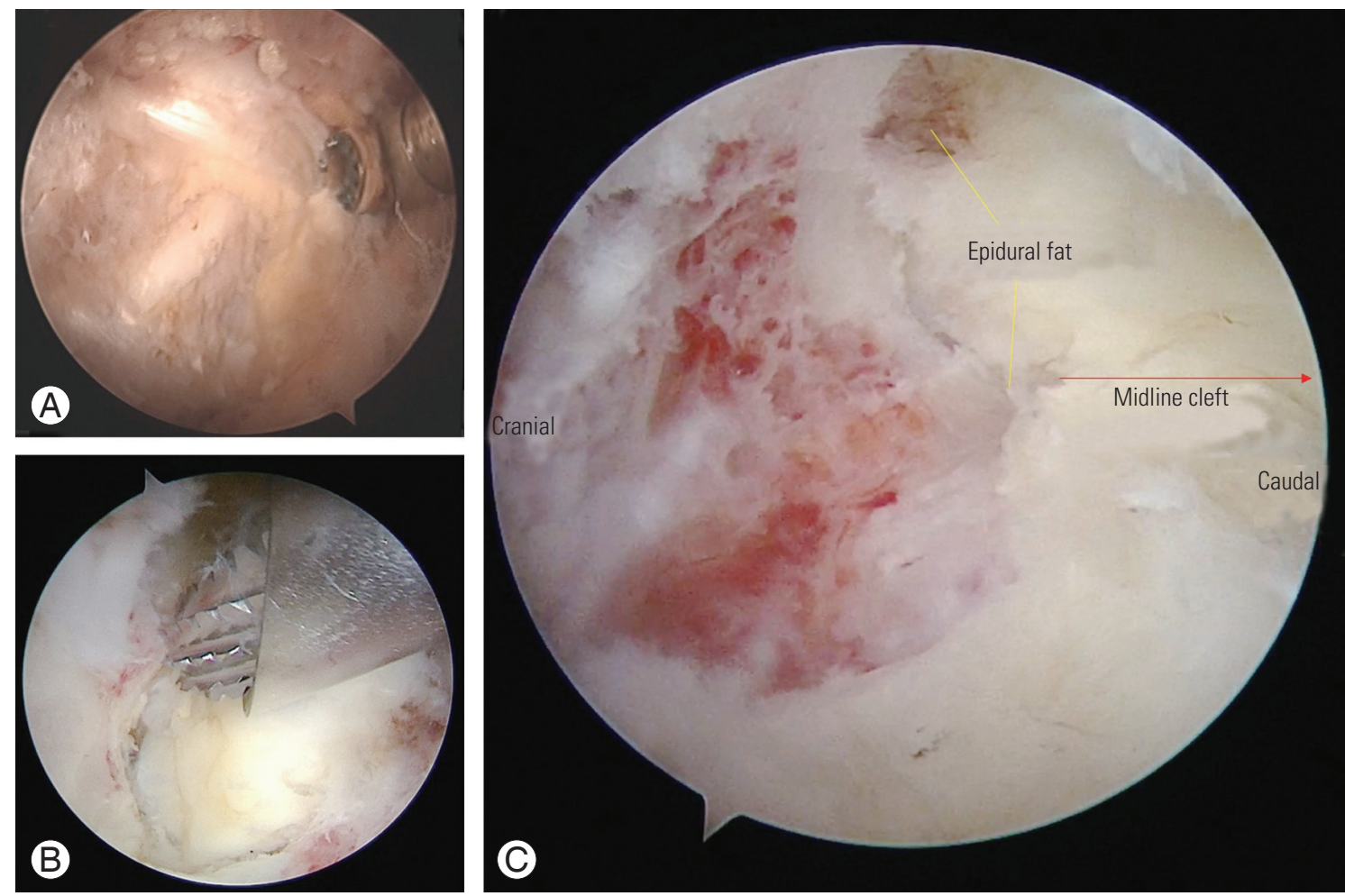

Fig. 3. (A) Exposure of spinolaminar junction and the interlaminar space. (B) The shielded burr and the intact flavum helps to protect the underlying dural structures. (C) Midline cleft identification. Epidural fat is seen at the most cranial border of the flavum origin.

margin of the contralateral flavum is reached (Fig. 3B, C). The cutting side of the burr should face away from the intact flavum that acts as a protective barrier for the underlying neural structures (Fig. 3B). The ipsilateral facet can be used as a fulcrum, levering away from the neurostructures to facilitate bone undercutting with burrs or Kerrison rongeurs. Flavum edges are then dissected off from the bone with a blunt dissector or a curette.

\section{9) Step 9}

Flavum midline is identified by central cleft and visualization of the epidural fat (Fig. 3B). Flavectomy starts from the midline toward the contralateral and from the cranial toward the caudal. The plane between the contralateral flavum and dura should be defined carefully using a blunt dissector and water influx. With scope trajectory following the dissecting plane, the contralateral approach offers a direct overhead view and instrumental access to the lateral recess instead of an inverted oblique view in the ipsilateral approach (Fig. 4A, B).

Before exposing the shoulder regions, direct flavectomy over the lateral recess can inadvertently cause nerve root injury. The shoulder region can be identified via the visu-
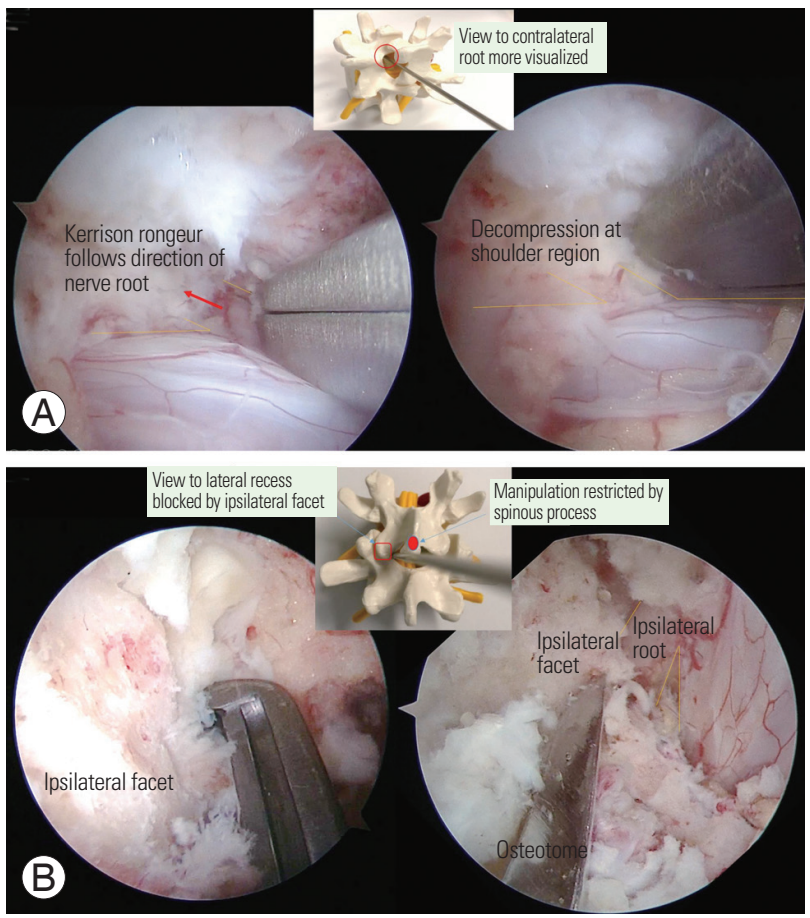

Fig. 4. (A) For contralateral approach, instrument insertion can follow the contralateral nerve root trajectory towards an anterior, lateral, and inferior direction (arrow). (B) Ipsilateral access can be limited due to pathologies like ipsilateral spinous process deviation and facet hypertrophy. Partial facetectomy may be required to expose the lateral recess. Instruments like osteotome can slip into the spinal canal when working on a vertically resected facet wall. 

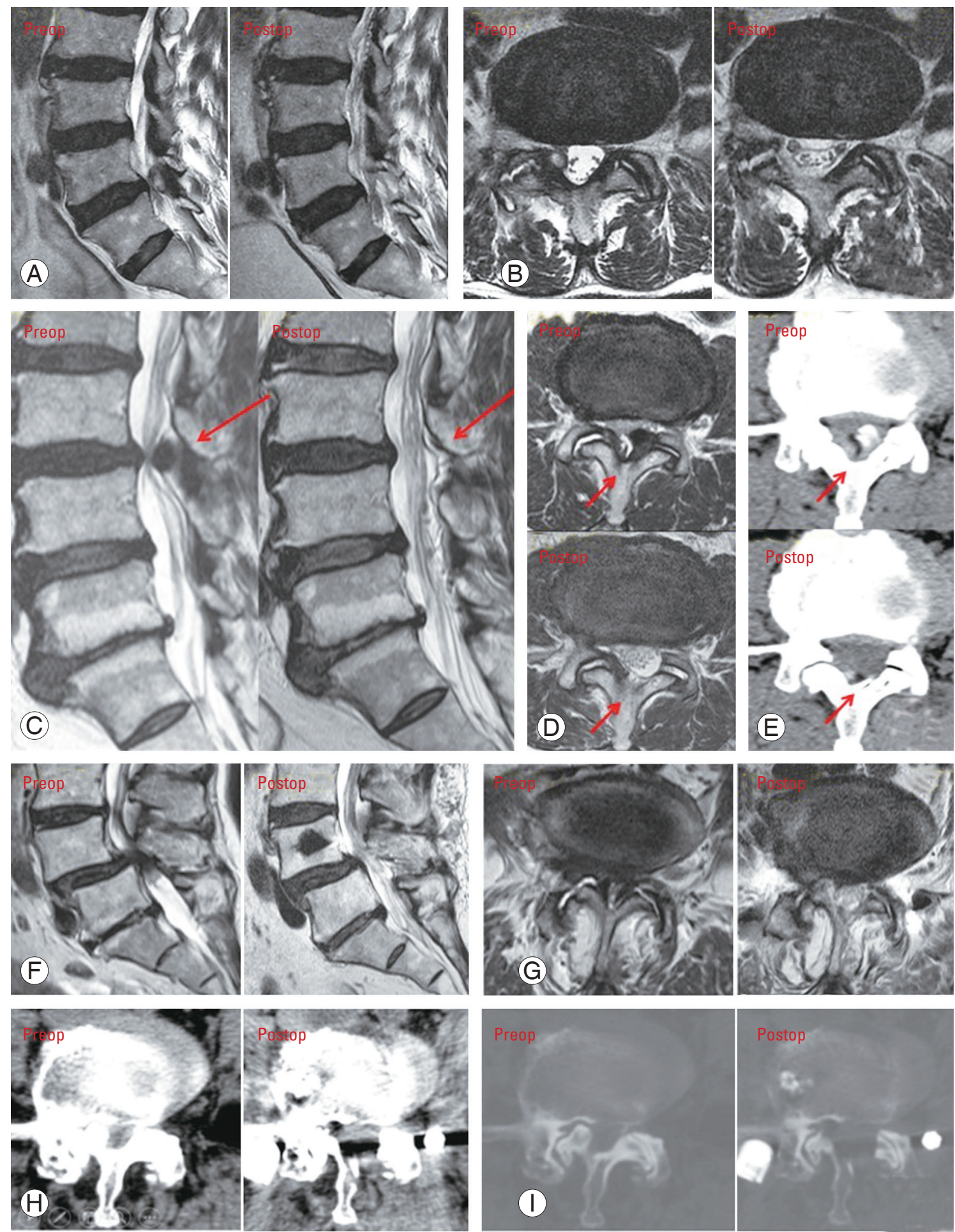

Fig. 5. (A-I) Radiographic illustration of three cases of asymmetric spinal stenosis, with bi-portal spinal decompression performed via contralateral approach. Preop, preoperative (before operation); postop, postoperative (after operation).

alization of epidural fat or whitish annulus over the lateral side of the root and pedicle palpation using a ball-tip probe. The transparent beveled cannula can then be rotat- ed to facilitate root retraction and exposure (Fig. 2C). In the case of paracentral or foraminal disc herniation, discectomy can be performed using small pituitary forceps 
and radiofrequency probe. Following decompression, a low-suction drain is inserted via the working portal under direct endoscopic visualization.

\section{Case illustrations}

\section{1) Case 1}

A 75-year-old man presented with a 1-year history of back pain that radiated to the right buttock, leg, and foot dorsum region. The pain aggravated when he stood up from the sitting position. The claudication distance was 5 minutes. The pain had worsened in recent months. Physical examination showed normal motor and sensory function in both the lower limbs. Preoperative magnetic resonance imaging (MRI) showed right L4/5 facet cyst, with compression on the $\mathrm{L} 5$ nerve root at the shoulder region (Fig. 5A, B).

Unilateral bi-portal endoscopic right L4/5 facetal cyst excision was performed via a left-sided approach. No dural tear was observed. Postoperatively, the patient experienced improvement in the right buttock and right foot dorsum pain. No wound or surgical complications were noted. The patient was discharged on postoperative day 8 .

On postoperative day 20, the pain over the right buttock radiating to the right leg and the foot reduced dramatically. The patient reported $100 \%$ pain relief in the right leg. At 1.5 months postoperatively, the patient reported an overall $90 \%$ reduction in back pain.

2) Case 2

A 73-year-old man who was premorbid walking unaided, complained of back pain, left hip pain, and weakness and numbness of left leg. Claudication distance was 5 minutes. The symptoms occurred since 5 years ago, becoming worse 2 months before our initial consultation (Fig. 5C-E).

The patient attended another hospital 1 month ago, diagnosed of L3/4 spinal stenosis. Back injection was given over the affected area, although the exact procedure could not be identified. Physical examination showed normal lower limb motor and sensory functions, except with diminished motor power of 4/5 for left ankle dorsiflexion. Preoperative computed tomography (CT) and MRI showed a 1.5-cm ossified ligamentum flavum at L3/4 level, causing left lateral recess stenosis and grade $\mathrm{C}$ central spinal stenosis.

Unilateral bi-portal endoscopic left L3/4 excision of the ossified flavum was performed via the right-sided approach. No dural tear was observed. No wound or surgical complications were noted. We observed rapid postoperative reduction in the left hip and buttock pain. Left ankle dorsiflexion improved to $4+/ 5$. The patient was able to walk independently with minimal postoperative pain. The patient was discharged on postoperative day 3. On the follow-up conducted at 2 weeks postoperatively, mild residual back pain and left leg pain was present. The patient was able to walk independently with minimal pain.

\section{3) Case 3}

A 69-year-old woman complained of right back and buttock tightness and right whole leg numbness, with radiating pain to the posterior thigh and calf. The claudication distance was 5 minutes. The condition manifested 5 years previously and had aggravated in the last month. The patient had attended another hospital previously and was diagnosed with L4/5 severe central stenosis and moderate right L4/5 foraminal stenosis. Nerve root injection was given without improvement. Examination showed normal lower limbs power and sensation. Numbness was present in particular the right foot region (Fig. 5F-I).

CT lumbar spine showed severe osteophytes at the right L4/5 facet joint, extending to the canal space. MRI lumbar spine showed concomitant broad-based right L4/5 bulging disc, causing grade-D central stenosis and obliteration of the right $\mathrm{L} 4 / 5$ lateral recess.

Unilateral bi-portal endoscopic decompression of the right L4/5 hypertrophic facet and spinal stenosis was performed using the left-sided approach. Additional dynamic screw (S-G) fixation was performed bilaterally at L4/5 levels. No dural tear was observed. No wound or surgical complications occurred. The patient's symptoms reduced from postoperative day, with reduced right buttock pain, numbness, and neurogenic claudication. Mild residual pain was present on the operation site and right buttock after patient-controlled analgesia was removed on postoperative day 3 . The patient's condition continued to improve throughout the in-hospital recovery period, and she could walk independently in the ward. She was discharged on postoperative day 12 . Her condition had stabilized at the 1-month follow-up after the surgery.

\section{Asymmetric pathologies: ipsilateral spinous process deviation and unilateral facet hypertrophy}

Van Schaik et al. [4] first described the following three types of spinous process deviations: (1) isolated deviation 


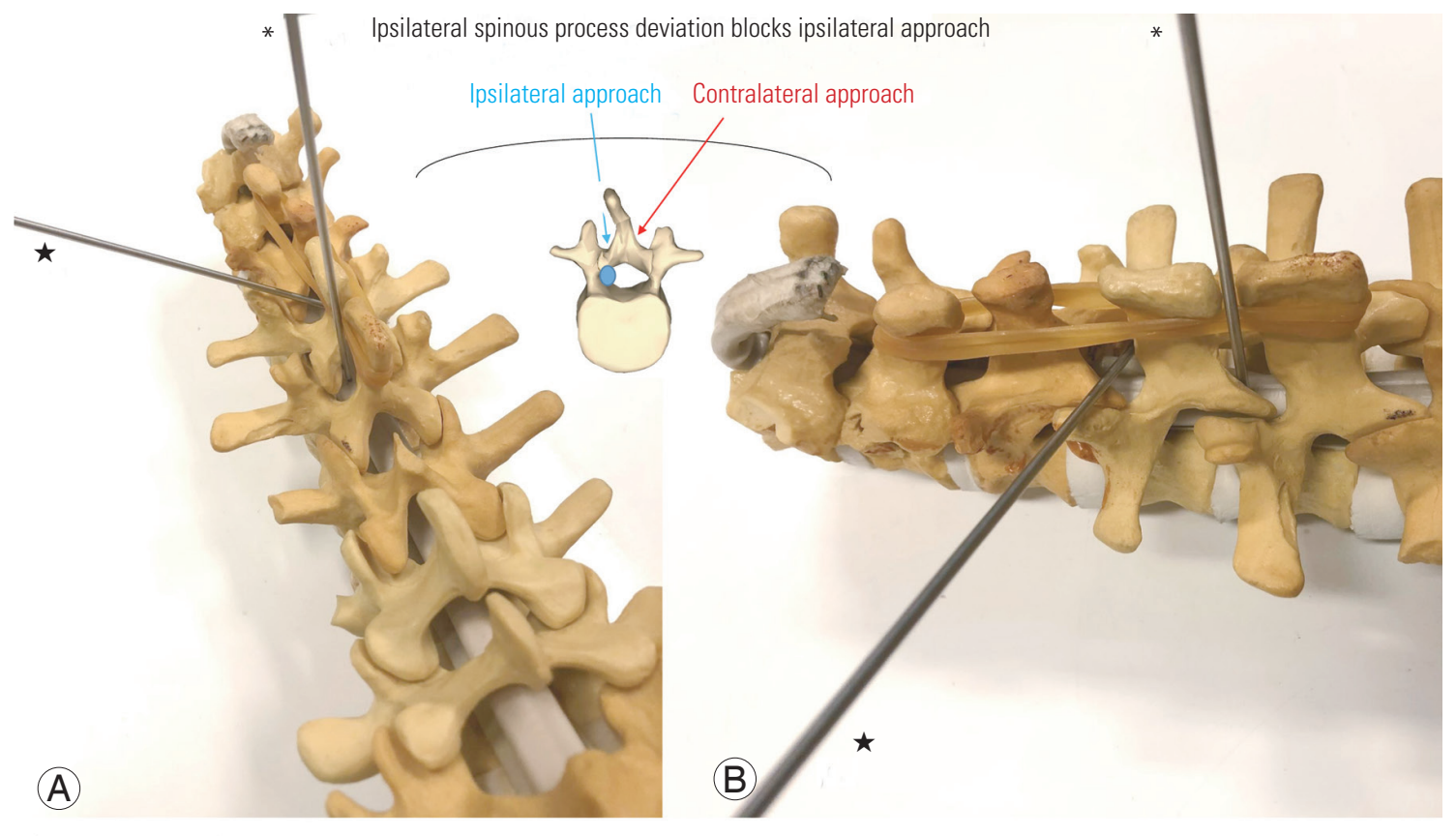

Contrateral approach provides a wider access in degenerative scoliosis
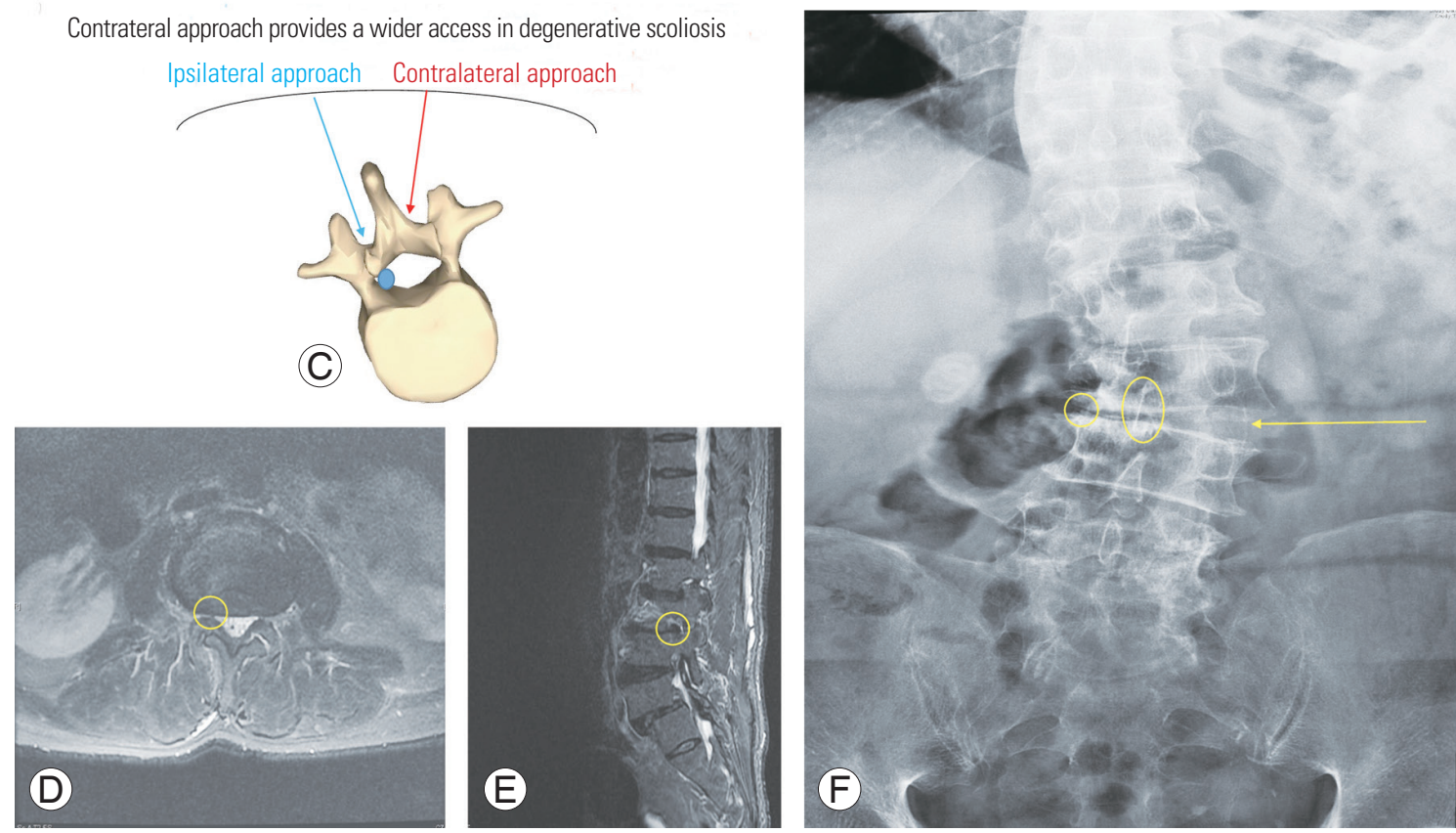

Fig. 6. (A) Ipsilateral access with a vertical trajectory may be blocked by an ipsilateral spinous process deviation, or limited by midline structures $\left({ }^{*}\right)$. (B) Contralateral access, with an oblique trajectory, may be less affected by bony constraints ( $\star$ ). (C) The relatively "open" corridor for the contralateral approach, when the spinous process is rotated to the concave side in degenerative scoliosis. (D-F) In the coronal dimension, the interlaminar space is wider on the convex side, theoretically being advantageous for portal entry via contralateral route.

of the spinous process, (2) rotatory scoliosis and degenerative arthritis, and (3) consequence of developmental dysplasia. Few studies have assessed the correlation of spinous process deviation and spinal degeneration. However, midline pathologies, including ossification of the interspinous ligament and ipsilateral spinous process deviation (Fig. 6A), or even the spinous process itself, may block the access via the ipsilateral route, assuming a more vertical trajectory (Fig. 6A). In contrast, approaching the contralateral recess will be less affected by midline bony constraints (Fig. 6B).

In degenerative scoliosis, vertebral rotation and lateral deviation of the spine occur together, with the rotation of spinous process directing toward the concavity of the 

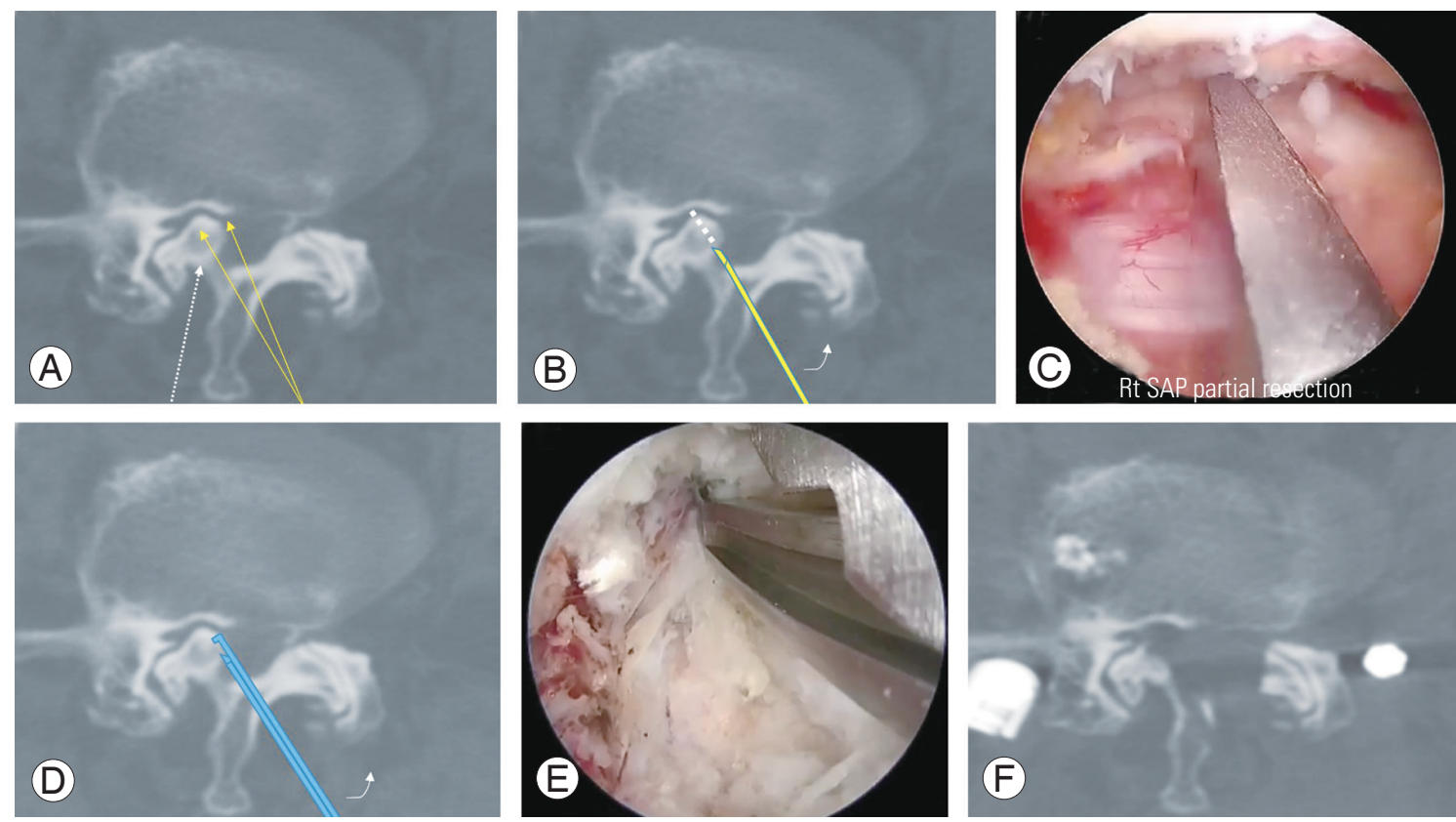

Fig. 7. (A) Solid and dotted arrows represent contralateral and ipsilateral routes respectively. Ipsilateral access is restricted by osteophytes overgrowth at the facet region. Contralateral access may be safer with plane dissection starting from normal anatomical structures. (B) Drawing of using osteotome to undercut the pathological facet joint, using the levering technique. (C) Endoscopic view of using osteotome to undercut the pathological facet joint, using the levering technique. (D) Drawing of using Kerrison to undercut the pathological facet joint, using the levering technique. (E) Endoscopic view of using Kerrison to undercut the pathological facet joint, using the levering technique. (F) Radiological outcome after operation. Rt SAP, right superior articular process.

curve. Radicular pain at the concave side can be caused by narrowed foramen or ruptured discs [5]. Madhavan et al. [6] described the phenomenon of nerve root compression at the concave side of the curve, leading to radicular pain. In reported series of Ploumis et al. [7] of 36 patients, $71.7 \%$ of radicular symptoms occurred on the concave side. For lateral stenosis arising on the concave side, the pathological anatomical structures are rotated deeper away from skin (Fig. 6C). In the axial dimension, the contralateral approach may offer a shorter skin-to-bone route and better spinous process clearance than with the ipsilateral approach. In the coronal dimension, the contralateral approach offers a wider interlaminar space for facilitating initial dissection, portal entry, and instrumental manipulation (Fig. 6D-F).

For unilateral facet hypertrophy, surgical decompression can be safely performed with partial undercutting facetectomy [8]. Farfan and Sullivan [9] were the first surgeons to discuss the relationship between discal and posterior articular abnormalities, addressing degenerative discal change, resulting in abnormal load transmission within the motion segment, and consequently causing unilateral facet osteoarthritis. In this group of patients, the ipsilateral route to lateral recess (dotted arrow) can be blocked by overriding osteophytes, and bone channel creation via the distorted anatomical structures may cause inadvertent damage to the neural elements (Fig. 7A). The contralateral approach (solid arrows) provides a safer corridor of entry between the bony and neural structures.

After determining the channel trajectory, a thin bone osteotome can be inserted to enlarge the working pathway (in yellow), following the planned plane of the hypertrophic facet undercutting (dotted line) (Fig. 7B). The osteotome can be levered away from the underlying neurostructures during hammering, while the dural sac should be constantly kept in sight to avoid accidental dural injury (Fig. 7C). Thereafter, a Kerrison laminectomy punch (in blue) can be inserted for further facet undercutting and decompression of the lateral recess (Fig. 7D). Similarly, the Kerrison punch can be levered away from dura that should be monitored endoscopically during undercutting (Fig. 7E). Fig. 7F shows the final radiological outcome following facet undercutting with the contralateral approach. 


\section{Discussion}

Unilateral bi-portal endoscopic spinal surgery has been rapidly evolving in recent years $[3,10,11]$. Compared to the instruments used for microscopic or full-endoscopic surgery, bi-portal endoscopic instruments may be less costly, while the learning curve is comparable. In standard open laminectomy, extensive muscle dissection and retraction may cause muscle denervation and necrosis [12], resulting in the loss of back extension power and dynamic spinal stability. This may cause postoperative back pain and muscle atrophy [13]. Endoscopic portal entry imposes limited surgical trauma even on the normal side because the portals are of limited size, and the starting point is over the spinolaminar junction that is devoid of muscle attachment and vascular supply. Bilateral decompression with the use of full endoscopic [14] and bi-portal endoscopic [10] methods for spinal stenosis have been described. However, to our knowledge, there is no clear description of the merits of unilateral decompression via the contralateral endoscopic approach.

The choice of contralateral or ipsilateral approach depends on each individual patient's spinal anatomy and pathology. In axial CT or MRI imaging, the mid-sagittal reference line is drawn through the center of the vertebral body or disk and the center of spinolaminar junction. It serves as the reference line for preoperative templating and measurement of the spinous process deviation and asymmetric lamina orientation [4], wherein the pathology may hinder ipsilateral access.

On the pathological side, various conditions can restrict both, viewing and access; for example, ossification of the interspinous ligament, facet arthropathy with overhanging osteophytes, ipsilateral spinous process deviation, and degenerative scoliosis with stenosis at the concave side. Approaching from the normal side may be technically easier with less pathological constraints and allows better anatomical identification. The plane between the dura and the pathological elements can be directly visualized from an overhead direction, using a zero-degree endoscope (Fig. 4A). Water pressure also aids in gentle plane dissection, softens flavum and adhesions, and provides a more spacious working space owing to the collapse of the dura. The normal anatomical plane is identified and opened up as the starting point, followed by gradual entry into the pathological zone. Dissection at the normal plane first is potentially safer with less chance of dural injury. In con- trast, initial plane separation on the pathological side first may be difficult, dural adhesions, and tightly compressed neuro-structures.

Preservation of the paraspinal muscle and facet joints, especially on the pathological side, is the most crucial consideration for non-fusion endoscopic spinal surgery. In our described contralateral approach, the paraspinal muscles and their bony attachments on the pathological side are relatively spared because the path of entry is from the normal side. The use of serial dilators can reduce the need for muscle stripping during the insertion of cylindrical portals. Moreover, the larger diameter and cylindrical shape design of the working portal helps in maximizing the contact surface area (Fig. 2A), dissipating the force generated from a small diameter instrumental device when it hinges on the portal. Compared to cylindrical cannulas, self-retaining retractors constitute the most significant factor for muscle injury in open spinal surgeries [12], leading to crush injury with elevated pressure and decreased perfusion. The injury severity is also associated with the pressure strength and retraction time, and muscle damage can be reduced by intermittent retractor release. Stevens et al. [15] showed that table-mounted tubular retractors produce lower retractor pressure in the surrounding tissues than self-retaining open retractors.

Leverage away from the neural structures may allow safer and more efficient bony undercutting, especially in pre-existing severe stenosis (Fig. 7C, E). However, there may be concerns regarding tissue retraction damage with the levering technique. We advocate a transverse incision, incising deeper crossing the medial fascia for cannula insertion and more superficial on the lateral margin, crossing the skin only for the preservation of the lateral fascia (Fig. 8A). This permits the instruments to be inserted at an oblique angle toward the contralateral side without excessive skin tension and retraction. As the working trajectory is oblique, the deeper structures will bear a smaller excursion force during leverage (Fig. 8B). The skin and the intact fascia preserved on the lateral wound margin will constitute the majority of the retraction forces, while the underlying multifidus muscle will be relatively spared. Nevertheless, the leverage time should be kept short with intermittent pressure release. The skin should be monitored at intervals to avoid pressure damage.

In a study of Ahn et al. [16] of unilateral laminotomy bilateral decompression bi-portal surgery, remarkable muscle signal changes were found on the immediate post- 

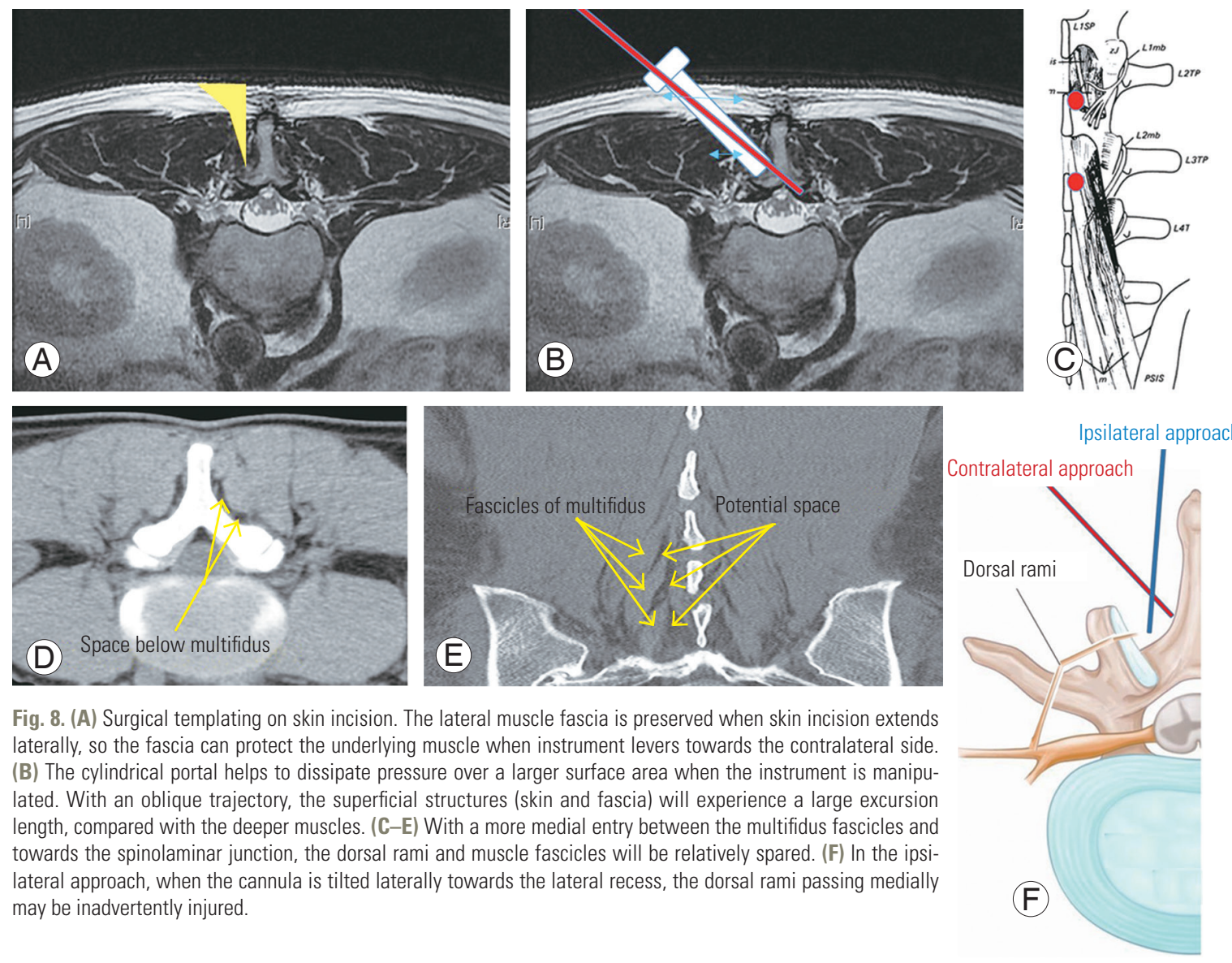

Fig. 8. (A) Surgical templating on skin incision. The lateral muscle fascia is preserved when skin incision extends laterally, so the fascia can protect the underlying muscle when instrument levers towards the contralateral side. (B) The cylindrical portal helps to dissipate pressure over a larger surface area when the instrument is manipulated. With an oblique trajectory, the superficial structures (skin and fascia) will experience a large excursion length, compared with the deeper muscles. (C-E) With a more medial entry between the multifidus fascicles and towards the spinolaminar junction, the dorsal rami and muscle fascicles will be relatively spared. (F) In the ipsilateral approach, when the cannula is tilted laterally towards the lateral recess, the dorsal rami passing medially may be inadvertently injured.

operative MRI scans on both, the ipsilateral and contralateral sides that correlated with the operation time. The signal intensity ratio (SIR) of the ipsilateral multifidus muscles increased up to $52 \%$ at the 2-week follow-up, compared with a $24.7 \%$ increase in the contralateral side. Furthermore, SIR of both the sides returned to baseline level at 4 weeks, achieving faster recovery than the muscle-preserving interlaminar decompression surgery in a study of Tonomura et al. [17]. There was no evidence of muscle atrophy, as shown by the changes in the crosssectional area.

The multifidus is particularly vulnerable to injury owing to monosegmental innervation from the medial branch of the dorsal rami. To further prevent injury to the multifidus muscle, the target landmark should be carefully designated. We recommend initial docking at the spinolaminar junction through the potential space between the multifidus fascicles that is relatively spared of nerve supply (Fig. $8 \mathrm{C}-\mathrm{E})$. In the ipsilateral approach, instrument directing laterally towards the lateral recess may lead to a higher risk of injury to the dorsal rami (Fig. $8 \mathrm{~F}$ ).
As the angle of visualization is limited in the ipsilateral approach, partial resection of the facet joint may be necessary to approach the lateral recess or the foramen. Matsumura et al. [18] concluded that the preservation of facet joint was $85.1 \%$ over the ipsilateral side and $95.9 \%$ over the contralateral side for L4/5 lumbar canal stenosis in microscopic decompression using tubular retractor. Similar findings were reported with unilateral full-endoscopic [19-21] and bi-portal endoscopic decompression [11], especially for upper lumbar levels with a narrow lamina [22]. For bi-portal ipsilateral decompression, because of the vertical trajectory, more of the outer superficial bone needs to be resected before the inner bone can be undercut to expose the lateral recess (Fig. 9). In bi-portal endoscopic contralateral decompression, preservation of the facet joint by undercutting of the superior articular process can be achieved without table tilting as opposed to microscopic decompression and is more tolerable than inferior facetectomy [19].

The therapeutic window of the unilateral bi-portal endoscopy resembles a three-dimensional hourglass model 

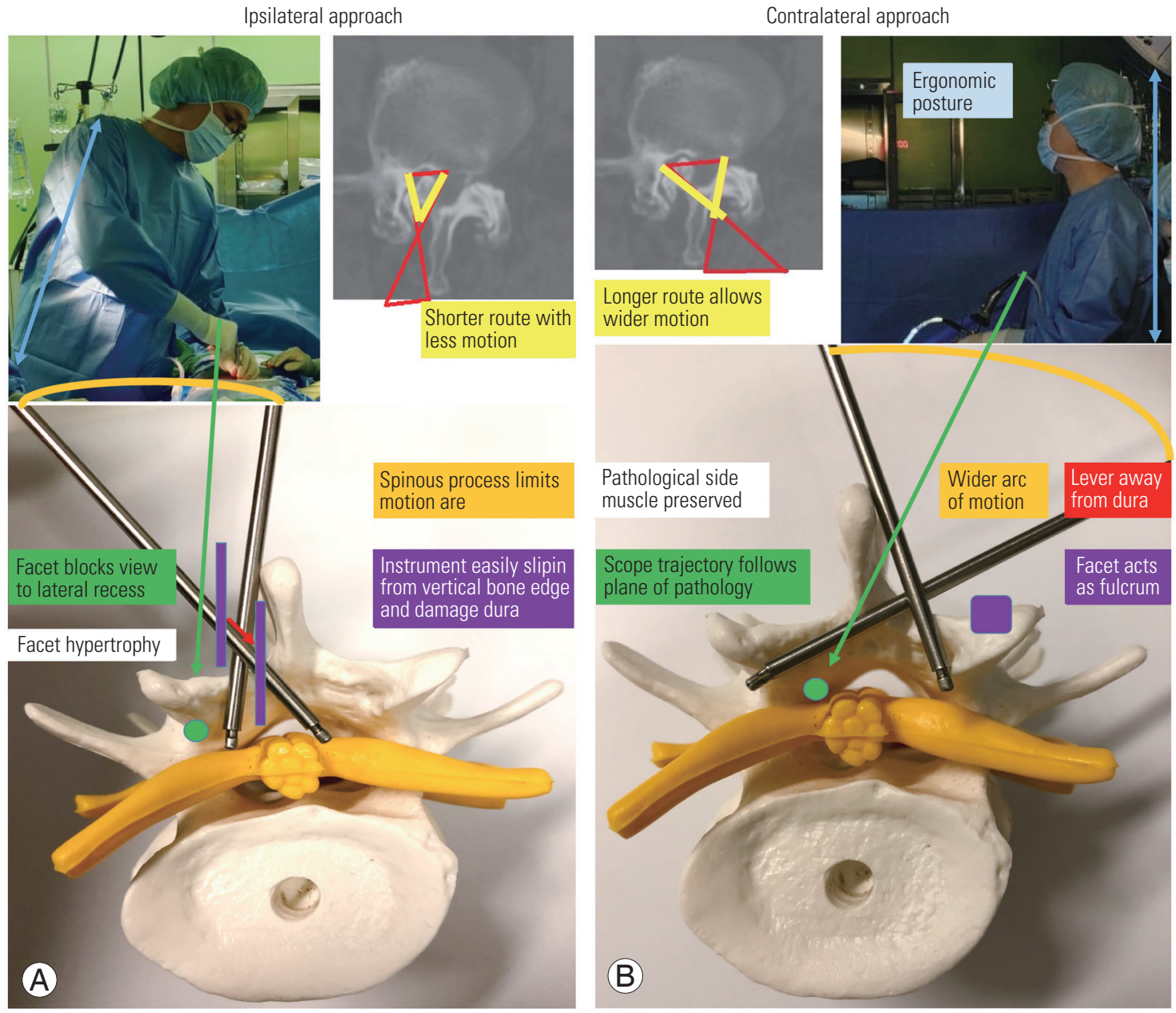

Fig. 9. (A, B) A schematic summary diagram illustrating comparison of contralateral versus ipsilateral approach in decompressing asymmetric spinal pathologies.

(Fig. 10). The outer cone is the cone of the working space, and its volume is directly proportional to that of the inner cone, aiding visualization and surgical intervention. While sagittal and coronal freedom is influenced by interlaminar width, motion in the transverse plane can be affected by anatomical barriers on the pathological side in asymmetric spinal stenosis as well as the spinous process. As the outer working space is less constrained over the normal side, the inner space for surgical intervention will be proportionally increased. The longer inner route to reaching pathology is advantageous because an extended moment arm indicates greater freedom of motion. The risks of neural injury may be reduced with a larger intervening space and a clear visual field.

The contralateral approach also offers an ergonomic position for surgeons. Surgeons can maintain an erect posture instead of leaning forward during the operation

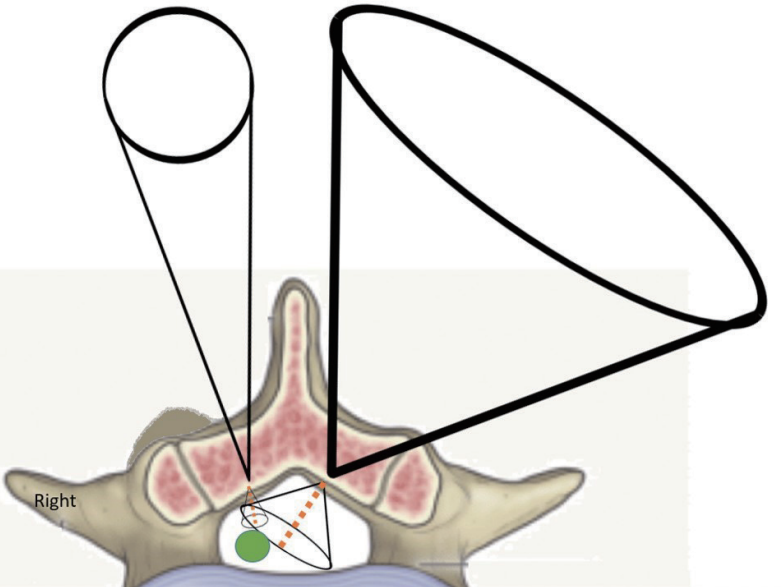

Fig. 10. The "hourglass theory". Right-sided ipsilateral approach is limited by hypertrophied facet and spinous process. Note also the longer inner route (dotted lines) in contralateral left-sided approach, which allows greater freedom of manipulation 
that reduces surgeon fatigue (Fig. 9). Decompression is achieved via leverage on the normal side of the facet joint, instead of working over an inverted facet edge. The placement of endoscopic instruments along a steep trajectory may also limit docking, and instruments can easily slip into the central spinal canal, damaging the dura (Fig. 9).

There are certain limitations associated with this approach, as in the case of central or extra-foraminal disc herniation or spinous process deviation towards the nonpathological side. Furthermore, cranial or caudal migrated discs require keyhole procedures to provide access to the desired level. Inadvertent pars fracture can be possible when surgical landmarks are lacking during contralateral sublaminoplasty. Although uncommon, dural tear repair can be more challenging than open surgeries.

\section{Conflict of Interest}

No potential conflict of interest relevant to this article was reported.

\section{References}

1. Gibson JN, Waddell G. Surgery for degenerative lumbar spondylosis: updated Cochrane review. Spine (Phila Pa 1976) 2005;30:2312-20.

2. Park WB, Hong JT, Lee SW, Sung JH, Yang SH, Kim IS. Clinical and radiological comparison between ipsilateral and contralateral side canal decompression using an unilateral laminotomy approach. Korean J Spine 2016;13:41-6.

3. Heo DH, Kim JS, Park CW, Quillo-Olvera J, Park CK. Contralateral sublaminar endoscopic approach for removal of lumbar juxtafacet cysts using percutaneous biportal endoscopic surgery: technical report and preliminary results. World Neurosurg 2019;122:4749.

4. Van Schaik JP, Verbiest H, van Schaik FD. Isolated spinous process deviation: a pitfall in the interpretation of AP radiographs of the lumbar spine. Spine (Phila Pa 1976) 1989;14:970-6.

5. De Vries AA, Mullender MG, Pluymakers WJ, Castelein RM, van Royen BJ. Spinal decompensation in degenerative lumbar scoliosis. Eur Spine J 2010;19:1540-4.

6. Madhavan K, Chieng LO, McGrath L, Hofstetter CP, Wang MY. Early experience with endoscopic forami- notomy in patients with moderate degenerative deformity. Neurosurg Focus 2016;40:E6.

7. Ploumis A, Transfeldt EE, Gilbert TJ, Mehbod AA, Pinto MR, Denis F. Radiculopathy in degenerative lumbar scoliosis: correlation of stenosis with relief from selective nerve root steroid injections. Pain Med 2011;12:45-50.

8. Getty CJ, Johnson JR, Kirwan EO, Sullivan MF. Partial undercutting facetectomy for bony entrapment of the lumbar nerve root. J Bone Joint Surg Br 1981;63B:330-5.

9. Farfan HF, Sullivan JD. The relation of facet orientation to intervertebral disc failure. Can J Surg 1967;10:179-85.

10. Kim JE, Choi DJ, Park EJJ, et al. Biportal endoscopic spinal surgery for lumbar spinal stenosis. Asian Spine J 2019;13:334-42.

11. Akbary K, Kim JS, Park CW, Jun SG, Hwang JH. Biportal endoscopic decompression of exiting and traversing nerve roots through a single interlaminar window using a contralateral approach: technical feasibilities and morphometric changes of the lumbar canal and foramen. World Neurosurg 2018;117:15361.

12. Kim CW. Scientific basis of minimally invasive spine surgery: prevention of multifidus muscle injury during posterior lumbar surgery. Spine (Phila Pa 1976) 2010;35(26 Suppl):S281-6.

13. Mobbs RJ, Li J, Sivabalan P, Raley D, Rao PJ. Outcomes after decompressive laminectomy for lumbar spinal stenosis: comparison between minimally invasive unilateral laminectomy for bilateral decompression and open laminectomy: clinical article. J Neurosurg Spine 2014;21:179-86.

14. Kim HS, Paudel B, Jang JS, et al. Percutaneous full endoscopic bilateral lumbar decompression of spinal stenosis through uniportal-contralateral approach: techniques and preliminary results. World Neurosurg 2017;103:201-9.

15. Stevens KJ, Spenciner DB, Griffiths KL, et al. Comparison of minimally invasive and conventional open posterolateral lumbar fusion using magnetic resonance imaging and retraction pressure studies. J Spinal Disord Tech 2006;19:77-86.

16. Ahn JS, Lee HJ, Park EJ, et al. Multifidus muscle changes after biportal endoscopic spinal surgery: magnetic resonance imaging evaluation. World Neu- 
rosurg 2019;130:e525-34.

17. Tonomura H, Hatta Y, Mikami Y, et al. Magnetic resonance imaging evaluation of the effects of surgical invasiveness on paravertebral muscles after musclepreserving interlaminar decompression (MILD). Clin Spine Surg 2017;30:E76-82.

18. Matsumura A, Namikawa T, Terai H, et al. The influence of approach side on facet preservation in microscopic bilateral decompression via a unilateral approach for degenerative lumbar scoliosis: clinical article. J Neurosurg Spine 2010;13:758-65.

19. Huang YH, Lien FC, Chao LY, Lin CH, Chen SH. Full endoscopic uniportal unilateral laminotomy for bilateral decompression in degenerative lumbar spinal stenosis: highlight of ligamentum flavum detachment and survey of efficacy and safety in 2 years of followup. World Neurosurg 2020;134:e672-81.
20. McGrath LB, White-Dzuro GA, Hofstetter CP. Comparison of clinical outcomes following minimally invasive or lumbar endoscopic unilateral laminotomy for bilateral decompression. J Neurosurg Spine 2019:1-9.

21. Ito F, Ito Z, Shibayama M, et al. Step-by-step sublaminar approach with a newly-designed spinal endoscope for unilateral-approach bilateral decompression in spinal stenosis. Neurospine 2019;16:41-51.

22. Kim HS, Patel R, Paudel B, et al. Early outcomes of endoscopic contralateral foraminal and lateral recess decompression via an interlaminar approach in patients with unilateral radiculopathy from unilateral foraminal stenosis. World Neurosurg 2017;108:76373. 